\title{
The effect of liquid viscosity on sliding friction coefficient of wet granular materials
}

\author{
Mojgan Aliasgari ${ }^{{ }^{*}}$, Nahid Maleki-Jirsaraei ${ }^{1}$, and Shahin Rouhani ${ }^{2}$ \\ ${ }^{1}$ Complex Systems Laboratory, Physics Department, Alzahra University, P.O. Box 1993891176, Tehran, Iran \\ ${ }^{2}$ Physics Department, Sharif University of Technology, P.O. Box 11155-9161, Tehran, Iran
}

\begin{abstract}
The mechanical properties of wet granular materials are different to those of dry granular matter. If the wet granular medium is not completely saturated, the capillary bridges form and the surface tension changes the elastic properties of the medium. We studied the sliding friction of a sledge over wet granular media and found that surface tension and viscosity have crucial effect on the dynamic friction coefficient of the wet granular media. Higher the viscosity of the interstitial liquid results in higher dynamic friction coefficient. Furthermore, viscous interstitial liquid shows time dependency behaviour. Viscosity makes the friction coefficient to increase since causes more the energy loss.
\end{abstract}

\section{Introduction}

Energy loss due to friction is a well-known characteristic of granular materials. The addition of a liquid can help to reduce this issue. Adding a small amount of water to a dry sand assembly results in a lower sliding friction coefficient in comparison with the dry system, However, by further increasing the amount of water, the friction coefficient goes up again $[1,2]$. The crucial effect here is that the liquid forms a capillary bridge between the grains, acting as a cohesive force, which results in a larger modulus and large angle of repose of a granular heap [3].

partly saturated wet granular media and saturated media show different behaviour; saturated systems easily flow and can say shows shear thinning flow but in unsaturated systems the capillary bridge holds the grains close each other and an integrated medium forms that cannot easily flow and shows less shear-thinning behaviour and strong tensile strength $[4,5]$. Besides, increasing the cohesion of the partially wet granular system leads to less shear-thinning behaviour [4]; viscosity of the interstitial liquid can affect the cohesion of such systems.

In most of the studies to date, the effect of the viscosity of the binder liquid is not considered, perhaps because authors usually use liquids with viscosity as low as water. Here, we perform experiments using viscous liquids to show how viscosity affects the sliding dynamic friction coefficient. In this paper, we study the effects of interstitial liquids, its surface tension and its viscosity on the sliding friction of wet granular media in an unsaturated regime. We measure the lateral force that resists the motion of a slider and calculate the dynamic coefficient friction as a function of the surface tension and viscosity of the interstitial liquid. We find that increasing the surface tension leads to a lower friction coefficient because the system becomes harder while viscosity leading to an increase it. An independent penetration hardness test was applied which confirms the friction response.

\section{Material and Methods}

In this study, we used sand (glass beads) with a grain size distribution, between $212 \mu \mathrm{m} 500 \mu \mathrm{m}(106 \mu \mathrm{m}$ and $212 \mu \mathrm{m})$. The full description of the friction test and penetration hardness experiment method is fully described in $[6,7]$. We first measured the lateral force versus time, F measured by the load cell when the slider moves with a constant velocity on the wet granular media, then we repeat this procedure for the granular media made by the different interstitial liquids(Table1).

Table 1. Liquids used in the experiments: $\phi$ is the weight percentage of glycerol to water $\eta$ is the viscosity; $\rho$ is the density; $\gamma$ is the surface tension $[8,9]$.

\begin{tabular}{cccc}
\hline \hline liquid & $\begin{array}{c}\gamma \\
(\mathrm{N} / \mathrm{m})\end{array}$ & $\begin{array}{c}\eta \\
(\mathrm{mPa} . \mathrm{s})\end{array}$ & $\begin{array}{c}\rho \\
\left(\mathrm{kg} / \mathrm{m}^{3}\right)\end{array}$ \\
\hline \hline $\begin{array}{c}\text { Water+8\% Laundry } \\
\text { detergent }\end{array}$ & 0.0300 & 1.005 & 1000 \\
$\begin{array}{c}\text { Water+5\% Laundry } \\
\text { detergent }\end{array}$ & 0.0307 & 1.005 & 1000 \\
$\begin{array}{c}\text { Water+3\% Laundry } \\
\text { detergent }\end{array}$ & 0.0311 & 1.005 & 1000 \\
$\begin{array}{c}\text { Water+1\% Laundry } \\
\text { detergent }\end{array}$ & 0.0316 & 1.005 & 1000 \\
$\quad$ Water & 0.0700 & 1.005 & 1000 \\
$\begin{array}{c}\text { water/glycerol } \\
\text { mixture( } \phi=0.5)\end{array}$ & 0.0700 & 6.00 & 1132 \\
$\begin{array}{c}\text { water/glycerol } \\
\text { mixture( } \phi=0.6)\end{array}$ & 0.0700 & 10.8 & 1159 \\
$\begin{array}{c}\text { water/glycerol } \\
\text { mixture( } \phi=0.65)\end{array}$ & 0.0700 & 15.2 & 1179 \\
$\begin{array}{c}\text { water/glycerol } \\
\text { mixture( } \phi=0.8)\end{array}$ & 0.0650 & 20 & 1212 \\
$\begin{array}{c}\text { Silicone Oil } \\
\text { Silicone Oil }\end{array}$ & 0.0197 & 5 & 915 \\
Silicone Oil & 0.0197 & 20 & 950 \\
Silicone Oil & 0.0197 & 350 & 1159 \\
\hline & 0.0197 & 1000 & 1179 \\
\hline & & \\
\hline
\end{tabular}

* Corresponding author: m.asgary@alzahra.ac.ir

A video is available at https://doi.org/10.48448/p9q7-3t04 


\section{Experiment and Results}

We investigate the effect of viscosity by applying water/glycerol mixture with various weight percentage $(\phi)$ and silicone base oils with different characteristic as interstitial liquids. The friction coefficient of a sledge sliding over a wet granular media defined as the friction force over the normal force.

Figure 1 shows that by increasing the interstitial liquid viscosity, the dynamic friction coefficient also increases. As the liquid viscosity increases, the capillary bridge becomes stronger, so that it requires more energy to break down the capillary bridges when a slider passes across the substrate made of a wet granular media. The results show that another factor other than the surface tension affects the systems response to the shear force, which increases the dynamic friction coefficient.

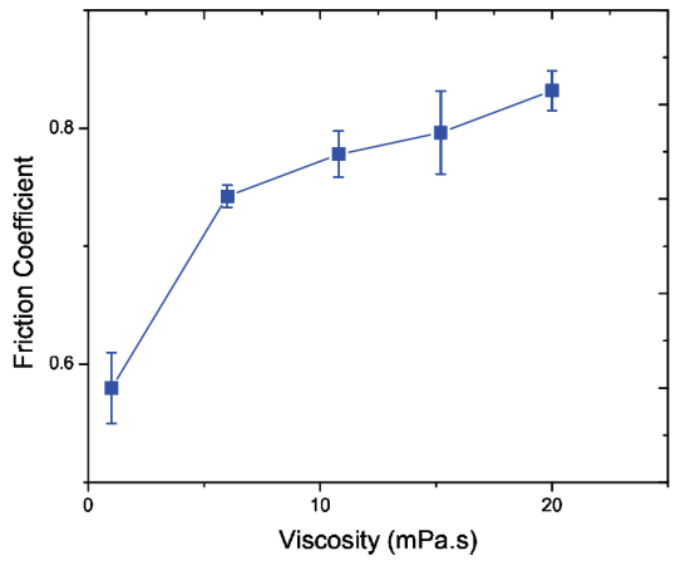

Fig. 1. Dynamic friction coefficient vs viscosity for water and glycerol mixture as the interstitial liquid in the sand. The volume fraction of the interstitial liquid (mixtures of pure water and glycerol) is fixed at $3 \%$ for all experiments. We use liquids with different viscosities but they have almost the same surface tension. As indicated in Table 1.

We repeated the above measurements using glass beads and silicone-based oils to have an interstitial liquid with higher viscosity. Although silicone oils have a higher viscosity in comparison with the water and glycerol mixtures they have lower surface tension. Needless to say, in experimental studies when you vary one parameter, also some others will change. The value for the viscosity and the surface tension of interstitial liquid are listed in Table 1. As we can see in figure 2 when surface tension is almost constant, dynamic friction increases with the viscosity. The silicone oils have surface tensions close to each other with the completely different viscosities, which leads to an increase in the friction coefficient of the wet granular system (glass beads and Silicone oil). The results show that the viscosity also affects the response of granular assembly to the shear force, in addition to the surface tension of the interstitial liquid.

Considering Ennis et al.'s theory [10], for viscous force:

$$
F_{v i s}=2 \pi R \frac{3 v \eta}{h}=2 \pi R \gamma \frac{3 C_{c a p}}{h}
$$

Where $\mathrm{v}$ is the relative velocity or compact velocity of the grains at impact, $\eta$ is the viscosity, $\gamma$ is the surface tension, $\mathrm{h}$ is the distance of the two beads connected by a liquid bridge and $C_{c a p}=v \eta / \gamma$ is the capillary number; the capillary number is a dimensionless number that describes the relative importance of viscous forces to capillary forces. It was shown that the influence of viscous forces increases linearly with the capillary number, and thus with the impact velocity (shear force) [11]. As it gradually increases, a transition from a regime dominated entirely by capillary force to a viscous regime takes place.

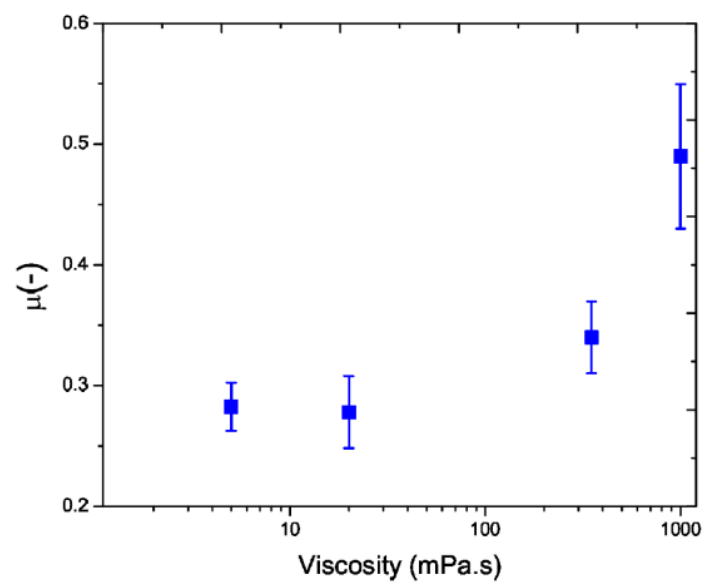

Fig. 2. Dynamic friction coefficient vs. viscosity, for different silicone oils as the interstitial liquid between glass beads. The values for the viscosities and surface tensions of the interstitial liquid are listed in Table 1. By increasing the viscosity, the friction coefficient increases too. We have increased the viscosity of the fluid with a constant levelling of surface tension. The results show that another factor other than fluid surface tension is effective on the system response to shear force, which increases the coefficient of friction dynamics.

To see the effect of velocity on the dynamic friction coefficient we test velocity dependency for two samples: (1) wetted sand with a mixture of pure water and glycerol $(\Phi=0.65$ with a viscosity of $15.2 \mathrm{mPa} . \mathrm{s})$ (2) pure water and sand mixture. All other parameters which affect the wet granular system such as the grain shape, grain radius distribution and the surface tension, viscosity is the only parameter varied.

As can be seen in Figure 3, in the low-viscosity case, the dynamic friction coefficient is independent of the velocity of the slider, as is expected, but for the highviscosity case the dynamic friction coefficient increases when the velocity of the slider increases. It has been proven that shear resistance increases with speed in granular media. However; partially saturated sand has much higher yield stress and should, therefore, have a much higher apparent viscosity in response to shear force [12]. when a slider moves over a wet granular matter, there is also a local flow of grains, it means a relative velocity between the grains, at high velocities of the slider, if the weight of the slider is high enough (when the normal force is high enough) then the viscose force becomes more important. These imply that for the liquids with a rather high viscosity, especially when the 
friction is rather high, we cannot ignore the effect of viscosity, which is in agreement with previous works [10-13]. Using viscous liquid like silicone-based oils as the interstitial liquid in the wet granular assembly can show velocity dependency conduct too.

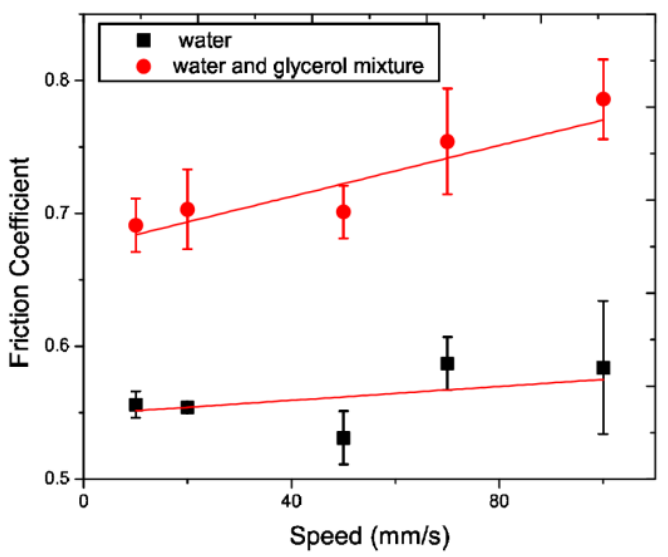

Fig. 3. Dynamic friction coefficient as a function of the velocity of the slider for two types of granular systems: sand + pure water (black solid squares) and sand + a water/glycerol mixture (weight percentage of 0.65 ; red solid circles). The volume fraction of the interstitial liquid is fixed at $3 \%$ for all experiments. Higher impact velocities result in more energy dissipation. The wet granular system with a high-viscosity liquid as the interstitial liquid shows higher friction coefficients and a stronger velocity-dependence.

Persson found a relation between the area of real contact and the load, which is consistent with the experiment results. Against the other theories of contact mechanics, he showed that the real contact area and load have a direct relation, $\Delta \mathrm{A}=\mathrm{L} / \mathrm{P}_{\mathrm{h}}$ where $\mathrm{L}$ is the load and $\mathrm{P}_{\mathrm{h}}$ (the penetration hardness) is the largest compressive stress that the materials can bear without plastic yielding. Coulomb's friction law is now easy to understand: the force $F$ necessary to shear junctions with total area $\Delta \mathrm{A}$ is given by $\mathrm{F}=\tau_{\mathrm{c}} \Delta \mathrm{A}$ where $\tau_{\mathrm{c}}$ is the yield stress during shear. The friction coefficient (defined by $\mathrm{F}=\mu \mathrm{L}$ ) can now be calculated:

$$
\mu=\frac{\tau_{c}}{P_{h}} \text {. }
$$

According to the Persson theory [14], capillary forces can affect the penetration hardness, as wet granular media can be more resistant against loads than dry ones, and the viscous forces affect the yield stress during shear.

When the slider leaves a trace in the sand after its passage [15], the granular medium is responding plastically rather than elastically and there is an inverse coloration between penetration hardness and friction coefficient [16]. The grains irreversibly move when a critical pressure, the penetration hardness $P_{h}$, is reached. Therefore, the sliding friction is controlled by the hardness of the material instead of the linear shear modulus.

We measure penetration hardness for silicone oil and glass beads assembly with different volume fractions of interstitial liquid, see Figure 4. As can be seen from the Figure 4 and Figure5, friction coefficient behaves inversely with penetration hardness.

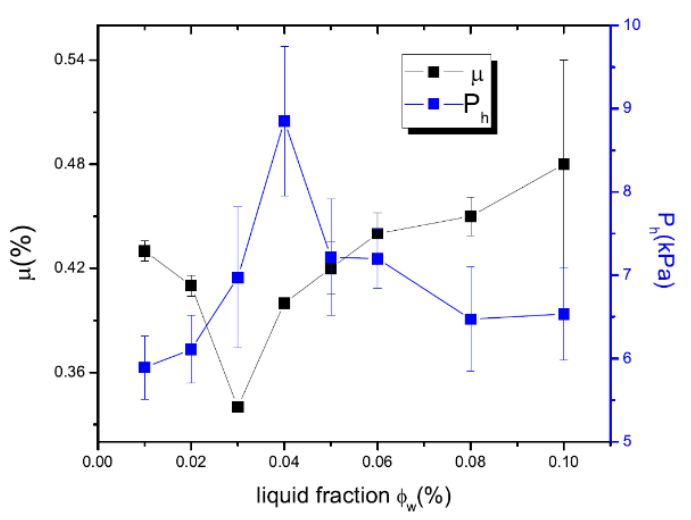

(a)

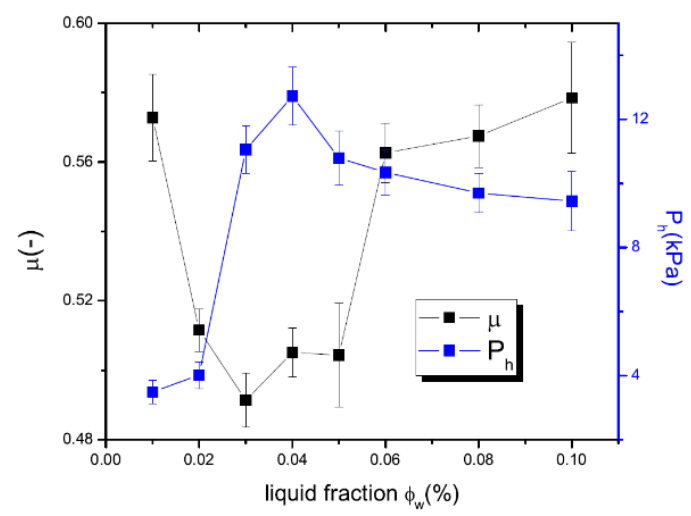

(b)

Fig. 4. Friction coefficient and penetration hardness vs. volume fraction of the interstitial liquid for glass beads and silicone oil mixture with (a) viscosity of $350 \mathrm{mPa} . \mathrm{s}$ (b) with viscosity of $1000 \mathrm{mPa}$.s. The size of the glass beads is between $106 \mu \mathrm{m}$ and $212 \mu \mathrm{m}$. the assembly with interstitial liquid with higher viscosity shows higher penetration hardness and friction coefficient. Above all of this we show that penetration hardness and friction coefficient act opposite each other. Stiffer media has low friction coefficient.

Moreover, the amount of friction at an optimum liquid fraction (here around 3\%) with a sample with higher viscosity (1000 mPa.s) is higher as well as $\mathrm{P}_{\mathrm{h}}$. This non-monotonic behaviour for wet glass beads is qualitatively in agreement with earlier measurements for wet sand $[1,10]$. In Figure 6 the penetration hardness almost increased regarding friction coefficient decreasing when $\mathrm{P}_{\mathrm{h}}$ normalized to viscosity it can be seen that all data is in a good agreement.

We employ wet sand with the water content of $3 \%$ and a glass beads and silicone oil assembly to compare the influence of the interstitial liquid with low (water) and high (silicone oil) viscosity. In this experiment, we cannot fix other factors (surface tension of the interstitial liquid, grain shape and size) since in the experiment when one parameter is varied also some others will change. Figure 6 reveals an increase in penetration hardness when the cone goes down faster through the wet glass beads. 


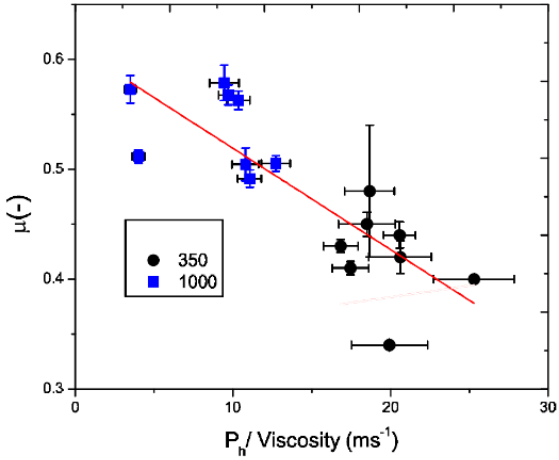

Fig. 5. Friction coefficient as a function of the penetration hardness which is normalized to viscosity. All were obtained as a function of the water volume fraction as given in Figure 4. For increasing hardness, a linear decrease of the friction coefficient is found; it becomes easier to slider over a hard and stiff glass beads- silicone oil mixture.

It can be concluded from Figure 6, the penetration hardness increases with the velocity when the viscosity of the interstitial liquid is high. In this study, the results show that another factor other than fluid surface tension is effective on the system response to shear force, which increases the dynamic friction coefficient. The results show that friction mechanism for wet granular systems is different from a completely solid system, which can be described by well-known friction theories (Amanton law and Bowden and Tabor theory [16])

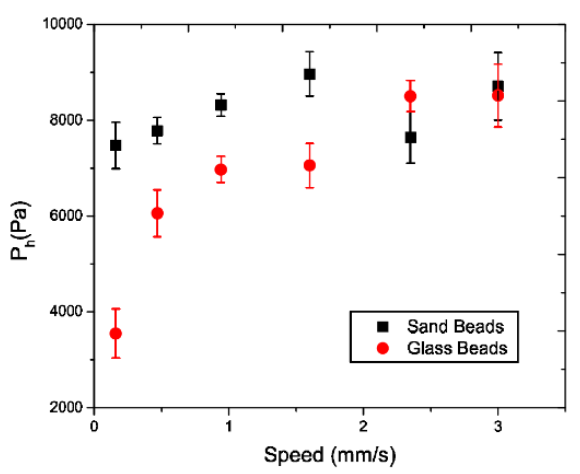

Fig. 6. Penetration hardness vs. speed of the cone for wet sand (black Solid Square) and wet glass beads (red solid circle). Silicone oil has the higher viscosity in comparison with water, see Table1, so at higher penetration speeds, the hardness of the glass beads and silicone oil assembly displays velocity dependence.

\section{Conclusion}

We studied the effect of viscosity and surface tension of interstitial liquids on the dynamic friction coefficient of wet granular media. Wet granular media can resist larger normal loads than dry ones because of the capillary force between grains created by the addition of interstitial liquid. This gives the wet granular matter elastic property. Earlier experiments were limited to low-viscosity liquids such as water and low-viscosity silicone oil [17]. It was shown that viscosity also plays a role in the dynamic mechanical properties of wet granular matter [18]. Therefore, we also addressed the question whether viscosity, which is a transport property, affects friction in wet granular materials. The coefficient of friction was previously shown to be related to the linear elastic modulus [1]. Our dynamic friction experiments on highly viscous liquids clearly reveal an effect of the viscosity on the friction coefficient.

This happens because surface tension makes capillary bridges stronger, which makes the elastic modules stronger and so helps the network to be more elastic in contrast to viscosity which makes the network more viscose and rigid. The slider's movement on the wet granular matter makes loses energy due to the deformation caused by higher viscose (and not elastic) bonds. In other words, increasing the surface tension decreases the friction coefficient by increasing the elasticity of network, and higher viscosity increases the friction coefficient because of the dissipative energy and deformation of a more rigid network.

\section{References}

[1] A. Fall, B. Weber, M. Pakpour, N. Lenoir, N. Shahidzadeh, J. Fiscina, C. Wagner, D. Bonn, Phys. Rev. Lett. 112, 175502 (2014)

[2] S. Karmakar, M. Schaber, A. L. Schuhmacher, M. Scheel, M. DiMichiel, M. Brinkmann, R. Seemann, L. Kovalcinova, L. Kondic, arXiv preprint arXiv: 1703. 08523 (2017)

[3] S. Roy, S. Luding, T. Weinhart, New J. Phys.19, 043014 (2017)

[4] S. Luding, Granul. Matter 10, 235246 (2008)

[5] O. Pitois, P. Moucheront, X. Chateau, J. Colloid Interface Sci. 231, 2631(2000)

[6] M. Aliasgari, N. Maleki-Jirsaraei, S. Rouhani, Z Phys Chem, (2019)

[7] R.W. Liefferink, M. Aliasgari, N. Maleki-Jirsaraei, S. Rouhani, D. Bonn, Granul. Matter 22, (2020)

[8] H.T. Chou, S.H. Chou, S.S. Hsiau, Powder Technol. 252, 4250 (2014)

[9] C.S. Miner, N.N. Dalton. American Chemical Society Monograph 1 (1953)

[10] B.J. Ennis, G.1. Tardos, R. Pfeffer, Powder Technol. 65, 257-272 (1991)

[11] G.D.R. Midi, Eur. Phys. J. E 14, 341-365 (2004)

[12] A.D. Salman, M. Hounslow, J.P.K. Seville, Granulation (Elsevier, Amsterdam, 2007)

[13] S. Herminghaus, Powder Technol. 54, 257-272 (2005)

[14] N. Persson, Sliding Friction: Physical Principles and Applications (Springer, 2000)

[15] R.W. Liefferink, B. Weber, D. Bonn, Phys. Rev. E 98, 052903 (2018)

[16] Q. Xu, A.V. Orpe, A. Kudrolli, Phys. Rev. E 76, 031302 (2007)

[17] P.C.F. Moller, D. Bonn, Europhys. Lett. 80, 38002 (2007)

[18] S.J.R. Simons, J.P.K. Seville, M.J. Adams, Chem. Eng. Sci. 49, 2331-2339 (1994) 